\title{
Expression and correlation of NRF2, KEAP1, NQO-1 and HO-1 in advanced squamous cell carcinoma of the larynx and their association with clinicopathologic features
}

\author{
CHANGJIANG LI ${ }^{1}$, HAITAO WU ${ }^{1}$, SHUYI WANG ${ }^{2}$ and JIAXING ZHU ${ }^{2}$ \\ ${ }^{1}$ Department of Otorhinolaryngology-Head and Neck Surgery, Shanghai Key Clinical Disciplines of Otorhinolaryngology; \\ ${ }^{2}$ Department of Pathology, Eye, Ear, Nose and Throat Hospital of Fudan University, Shanghai 200031, P.R. China
}

Received October 1, 2015; Accepted October 13, 2016

DOI: $10.3892 / \mathrm{mmr} .2016 .5913$

\begin{abstract}
The present study aimed specifically investigate the expression and correlation of kelch-like ECH-associated protein-1 (KEAP1), nuclear factor (erythroid-derived 2)-like 2 (NRF2), quinone oxidoreductase-1 (NQO-1) and heme oxygenase-1 (HO-1) in laryngeal cancer and their association with clinicopathological features. A total of 33 paired human fresh advanced laryngeal cancer and adjacent normal specimens were collected following total laryngectomy. Immunohistochemical and immunoblotting analysis were performed to evaluate the expression of KEAP1, NRF2, NQO-1 and HO-1. All of the patients clinicopathologic features were collected. Immunohistochemistry indicated that NRF2-positive staining was as high as $79 \%$ (26/33) in the cancer samples and predominantly located in the nuclei, whereas positive expression of KEAP1, NQO-1 and HO-1 was detected in 70\% (23/33), $76 \%$ (25/33) and 82\% (27/33) of cancer tissues, respectively, and primarily expressed in the cytoplasm. The corresponding adjacent normal samples produced almost no or weak expression of the proteins. There were significant differences in protein expression between the two groups $(\mathrm{P}<0.01)$. Immunoblotting analysis also demonstrated that their expression levels were higher in cancer tissue compared with adjacent normal tissue. Notably, KEAP1, NQO-1 and HO-1 expression was positively correlated with nuclear NRF2 in cancer tissues, whereas they had no correlation with age, tumor stage (clinical stage III and IV), tumor size and lymph node metastasis. In conclusion, increased expression of NRF2, KEAP1, NQO-1 and HO-1 were common in advanced laryngeal cancer. Evaluation of
\end{abstract}

Correspondence to: Professor Haitao $\mathrm{Wu}$, Department of Otorhinolaryngology-Head and Neck Surgery, Shanghai Key Clinical Disciplines of Otorhinolaryngology, Eye, Ear, Nose and Throat Hospital of Fudan University, 83 Fenyang Road, Shanghai 200031, P.R. China

E-mail: eentwuhaitao@163.com

Key words: advanced laryngeal squamous cell carcinoma, KEAP1, NRF2, NQO-1, HO-1 these factors may have important clinical significance for the diagnosis and treatment of this disease.

\section{Introduction}

Annually, 500,000 people are diagnosed with laryngeal cancer worldwide (1). The prevalence of laryngeal cancer differs widely between different geographic areas. In Europe, $\sim 52,000$ new cases are diagnosed annually, and an estimated 13,560 new cases appear in the United States (2). The yearly incidence ratio is $\sim 1.4 / 100,000$, which is standardized in China (3). Laryngeal cancer accounts for 2-3\% of all cancer and $25 \%$ all head and neck cancer. The majority (90\%) of laryngeal cancers occur in men, and $95 \%$ are squamous cell carcinoma, which is the second most common head and neck squamous cell cancer. Patients have a good prognosis when laryngeal cancer is discovered and treated timely. At the initial evaluation, $\sim 40 \%$ of patients exhibit stage III or IV disease (4). Although the 5-year overall survival rate has risen for the last 30 years due to intensive research and advances in conventional cancer treatments, terminal laryngeal cancer remains a troublesome problem for otolaryngologists. Thus, it is necessary to develop novel therapies to diagnose this cancer early and to rapidly treat the disease.

Certain studies have demonstrated that laryngeal cancers are closely associated with oxidative stress $(5,6)$. The body has developed a system to scavenge oxidants in response to harmful stimulations. Recent studies identify the role of the kelch-like ECH-associated protein-1 (KEAP1)/nuclear factor (erythroid-derived 2)-like 2 (NRF2) system in this process, and these proteins have central functions in cellular protection against oxidative stress. Under normal conditions, NRF2 couples with the anchor protein KEAP1 in the cytoplasm via Cul3-based E3 ligase, which is a negative regulator of NRF2 and promotes continuous degradation of NRF2 via ubiquitination and proteasomal degradation systems. NRF2 separates from KEAP1 following exposure to oxidative stress and translocates to the nucleus for the transactivation of AU-rich element (ARE)-bearing genes and phase II detoxifying enzymes via heterodimerization with small Maf proteins. Evidence indicates that the loss of KEAP1 genes leads to NRF2 upregulation and expression of its downstream genes 
that encode cytoprotective proteins, including quinone oxidoreductase-1 (NQO-1) and heme oxygenase-1 (HO-1), which enhances cancer cell proliferation. Simultaneous knockdown of NRF2 genes produced the opposite results. Experiments confirmed this hypothesis in lung and colorectal cancer (7-9).

To the best of our knowledge, no individual study has investigated the expression and correlation of NRF2, KEAP1, NQO-1 and HO-1 and their associations to clinicopathological features in advanced laryngeal cancer. Thus, the present study primarily focused on these indicators in a series of laryngeal cancer and paraneoplastic specimens to improve clinical diagnosis and treatment of advanced laryngeal cancer.

\section{Materials and methods}

Tissue samples. The Animal Ethical Committee of the Eye, Ear, Nose and Throat Hospital of Fudan University (Shanghai, China) reviewed and approved the study protocol. Specimens for this study were collected from 40 patients at the Otorhinolaryngology Head and Neck Surgery Department of the Eye, Ear, Nose and Throat Hospital of Fudan University from April 2014 to January 2015. Five cases were excluded because the tumor cells infiltrated the pericarcinous tissues. All patients were diagnosed with stage III or IV laryngeal cancer (10), except for two cases of stage II cancer. Samples of laryngeal cancer and adjacent normal tissues were collected from a total of 33 patients with clinical stage III or IV. All patients were male with an age range from 44 to 81 years (mean 61.7 \pm 7.6 years old). No patients had received any prior treatment for cancer, and there were no other systemic complications, including heart, hepatic or renal disease. All tumors were moderate differentiated. All patients underwent primary total laryngectomy. All surgical excisions of fresh specimens from the larynx neoplasm and adjacent tissues, which were $>1.5 \mathrm{~cm}$ away from the cancer, were used for immunohistochemistry and western blotting. Two pathologists confirmed that all tumor tissues were laryngeal squamous cell carcinomas, and all pericarcinous tissues were confirmed as normal tissues. The clinicopathological features are summarized in Table I.

Immunohistochemistry. The immunohistochemistry method used was reported previously (11). The 33 tumor and pericarcinomatous specimens were fixed in $4 \%$ paraformaldehyde and embedded in paraffin following routine processing. Sections ( $4 \mu \mathrm{m}$ thick) were obtained from each paraffin block. The sections were cleared in xylene and hydrated through a descending alcohol series to distilled water. Endogenous peroxidase activities were quenched with $3 \%$ hydrogen peroxide in deionized water for $10 \mathrm{~min}$. An antigen retrieval step was performed using citrate buffer, $\mathrm{pH} 6.0$, for $10 \mathrm{~min}$, and sections were brought boiled for $10 \mathrm{~min}$ in two 5 -min sessions in a microwave. Sections were preincubated with $1 \%$ normal bovine serum (Gibco; Thermo Fisher Scientific, Inc., Waltham, MA, USA) to block nonspecific binding sites for $1 \mathrm{~h}$ at room temperature and incubated overnight at $4^{\circ} \mathrm{C}$ with anti-NRF2/KEAP1/HO-1 antibodies [rabbit polyclonal to NRF2 (cat. no. ab31163), rabbit monoclonal to KEAP1 (cat. no. ab181144) and HO-1 (cat. no. ab52947); Abcam, Cambridge, UK] at a 1:100 dilution or an NQO-1 antibody
Table I. Clinicopathological features of patients with laryngeal cancer.

\begin{tabular}{lc}
\hline Feature & $\begin{array}{c}\text { Number of } \\
\text { cases }\end{array}$ \\
\hline Total & 33 \\
Age & \\
$\geq 62$ years old & 19 \\
$<62$ years old & 14 \\
Sex & \\
Male & 33 \\
Female & 0 \\
Differentiation & \\
G1 & 5 \\
G2 & 28 \\
G3 & 0 \\
Clinical stage & \\
III & 16 \\
IV & 17 \\
Lymph node metastasis & \\
N0 & 13 \\
N+ & 20 \\
Maximum diameter of tumor size & \\
$\geq 3.2 \mathrm{~cm}$ & 17 \\
$<3.2 \mathrm{~cm}$ & 16 \\
\hline
\end{tabular}

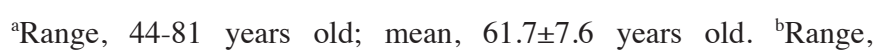
$1.6-5.5 \mathrm{~cm}$; average, $3.2 \mathrm{~cm}$.

[mouse monoclonal to NQO-1 (cat. no. 3187); Cell Signaling Technology, Inc., Danvers, MA, USA] at a 1:25 dilution. The primary antibody was only added to one of the two sections on each slide, and the other section was incubated with PBS as a control. The slides were washed with PBS and incubated with the secondary antibody (part of ABC complex kit) for $15 \mathrm{~min}$ at $37^{\circ} \mathrm{C}$, followed by incubation with the $\mathrm{ABC}$ complex kit (Wuhan Boster Biological Technology, Ltd., Wuhan, China). Color development and visualization was performed using 3,3-diaminobenzidine. The tissue sections were lightly counterstained with hematoxylin, dehydrated, cleared and coverslipped. The slides were imaged using a light microscope.

Immunohistochemical semiquantitative evaluations of cytoplasmic and nuclear proteins were performed separately. An immunohistochemical expression score ( 0.0 to 4.0 scoring system) was used, and a final score was obtained by multiplying the percentage of stained cells and corresponding intensity score. A score of 0 suggested that there was no detectable staining and 4.0 corresponded to a saturated signal in the tissues. An immunostaining score $\geq 0.5$ was chosen arbitrarily as overexpression of cytoplasmic proteins, and positive expression of nuclear proteins was defined as a score $\geq 0.1$ based on the associated literature (12). This method of deriving immunoscores was reported in numerous previous studies $(12,13)$. Two pathologists evaluated all immunostaining in a blinded 

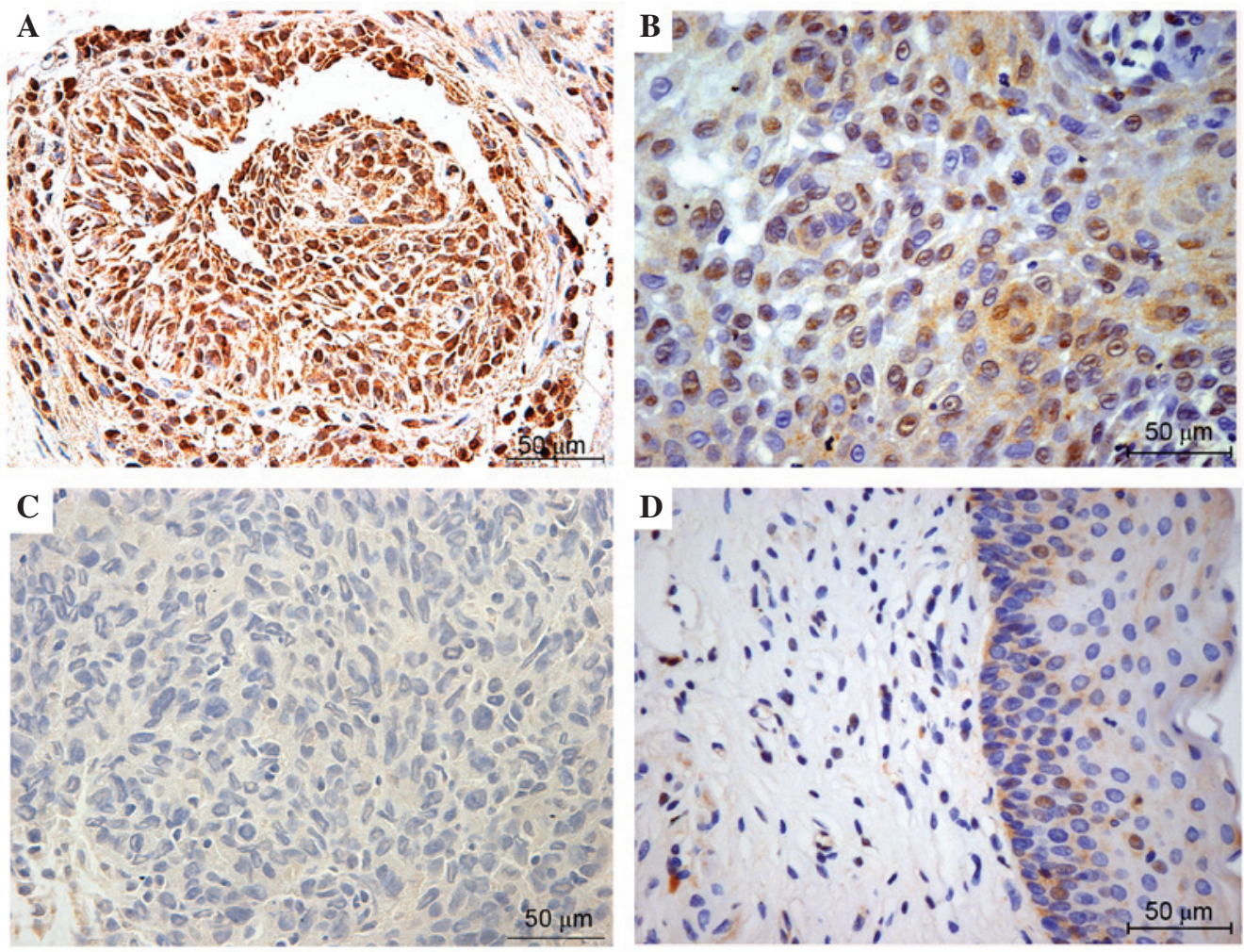

Figure 1. Representative immunohistochemical staining of NRF2 in laryngeal squamous cell carcinoma and adjacent normal mucosa. Top images exhibit (A) strong and (B) positive expression of NRF2, which is primarily located in cancer cell nuclei. The bottom images exhibit negative NRF2 expression in (C) laryngeal cancer and (D) pericarcinomatous tissues. Positive nuclear NRF2 was defined as a score $\geq 0.1$ (magnification, $x 40$ ). NRF2, nuclear factor (erythroid-derived 2)-like 2.

manner, without any clinical information. Negative controls of nonspecific immunohistochemical staining were routinely included. A total of 3 randomly selected fields from each sample were examined.

Western blot analysis. All frozen tissues were lysed in a radioimmunoprecipitation buffer containing phenylmethylsulfonyl fluoride with a final concentration of $1 \mathrm{mM} / 1$ (CoWin Biotech Co., Ltd., Beijing, China), and total protein extracts were obtained using centrifugation at $4{ }^{\circ} \mathrm{C}$ and $10,000 \times \mathrm{g}$ for $10 \mathrm{~min}$. Protein concentration was quantified using a Bicinchoninic Acid Protein Assay kit (Beyotime Institute of Biotechnology, Haimen, China), and $40 \mu \mathrm{g}$ total protein were electrophoretically separated on $12 \%$ sodium dodecyl sulfate polyacrylamide gel, transferred to polyvinylidene fluoride membranes, which were blocked with 5\% non-fat milk in Tris-buffered saline with $0.1 \%$ Tween 20 (TBST; $\mathrm{pH}=7.4-7.5$ ) at room temperature for $1 \mathrm{~h}$. Membranes were incubated overnight with anti-NRF2 (cat. no. ab31163), anti-KEAP1 (cat. no. ab181144), anti-NQO1 (cat. no. 3187), anti-HO1 (cat. no. ab52947) or anti- $\beta$-actin (cat. no. AA128; Beyotime Institute of Biotechnology) primary antibodies in blocking buffer at $4^{\circ} \mathrm{C}$ at a $1: 1,000$ dilution. Membranes were washed three times in TBST followed by incubation for $1 \mathrm{~h}$ with a horseradish-peroxidase goat anti-rabbit (cat. no. A0208; Beyotime Institute of Biotechnology) and goat anti-mouse (cat. no. A0216; Beyotime Institute of Biotechnology) secondary antibody at a 1:2,500 dilution. Membranes were washed in TBST, and bands were visualized using enhanced chemiluminescence kit (beyoECL
Plus; Beyotime Institute of Biotechnology). $\beta$-actin was used as the internal control.

Statistical analysis. The significance of intergroup differences in NRF2, KEAP1, NQO-1, and HO-1 immunostains between laryngeal cancer and pericarcinomatous specimens were determined using Fisher's exact test. Mutual correlations on the protein expression levels and their association with clinicopathological features in carcinoma tissues were analyzed using Pearson correlation test and independent-samples $t$ test respectively, prior to which normality test was applied. The degree of correlation was determined using Pearson correlation coefficient. $\mathrm{P}<0.05$ (two-sided) was considered to indicate a statistically significant difference. All statistical analyses were conducted using the SPSS 17.0 statistical package (SPSS, Inc., Chicago, IL, USA). All of the statistical graphs were produced using with GraphPad Prism 5.0 (GraphPad Software, Inc., La Jolla, CA, USA).

\section{Results}

Immunohistochemical detection of NRF2 (Fig. 1), KEAP1 (Fig. 2), NQO-1 (Fig. 3) and HO-1 (Fig. 4) activities assessed the expression and localization of these proteins in laryngeal carcinoma samples. High expression levels of NRF2 were common in advanced laryngeal cancer tissue, and expression was primarily localized in the nuclei of these specimens. NRF2 was weakly expressed in the cytoplasm of the tumor cells. In non-neoplastic samples, NRF2 was barely expressed. 

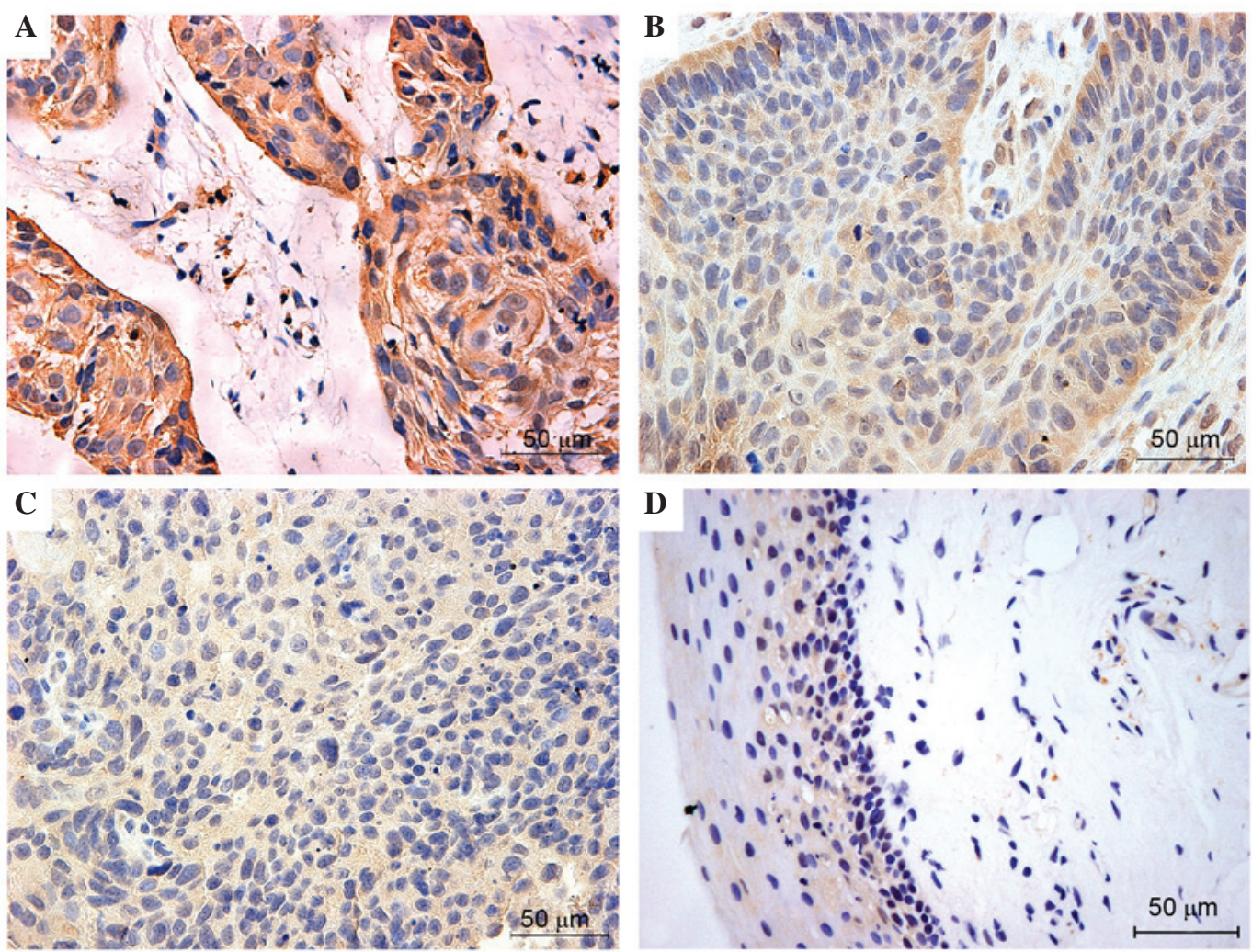

Figure 2. Representative immunohistochemical staining of KEAP1 in laryngeal squamous cell carcinoma and adjacent normal mucosa. The top two images exhibit (A) strong and (B) positive expression of KEAP1 in the cancer cell cytoplasm. The bottom two images exhibit negative KEAP1 expression in (C) laryngeal cancer and (D) pericarcinomatous tissues. Normal mucosa produced almost no positive KEAP1 expression. Positive cytoplasmic KEAP1 was defined as a score $\geq 0.5$. KEAP1, kelch-like ECH-associated protein-1.
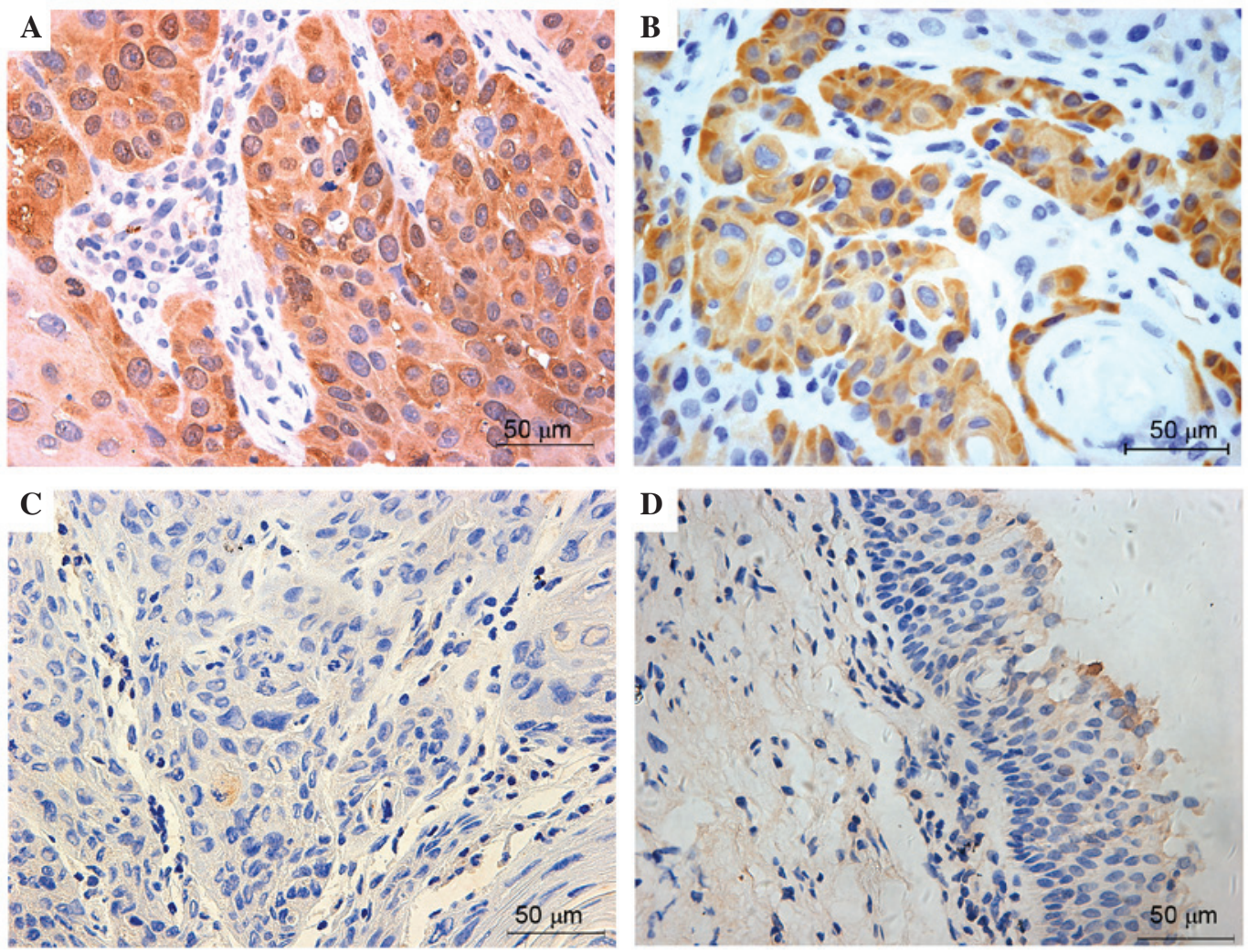

Figure 3. Representative immunohistochemical staining of NQO-1 in laryngeal squamous cell carcinoma and adjacent normal mucosa. The top two images exhibit (A) strong and (B) positive expression of NQO-1, which was primarily observed in the cancer cell cytoplasm. The bottom two images exhibit negative NQO-1 expression in (C) laryngeal cancer and (D) pericarcinomatous tissues. Normal mucosa produced almost no positive NQO-1 expression. Positive cytoplasmic NQO-1 was defined as a score $\geq 0.5$ (magnification $x 40$ ). NQO-1, quinone oxidoreductase- 1 . 

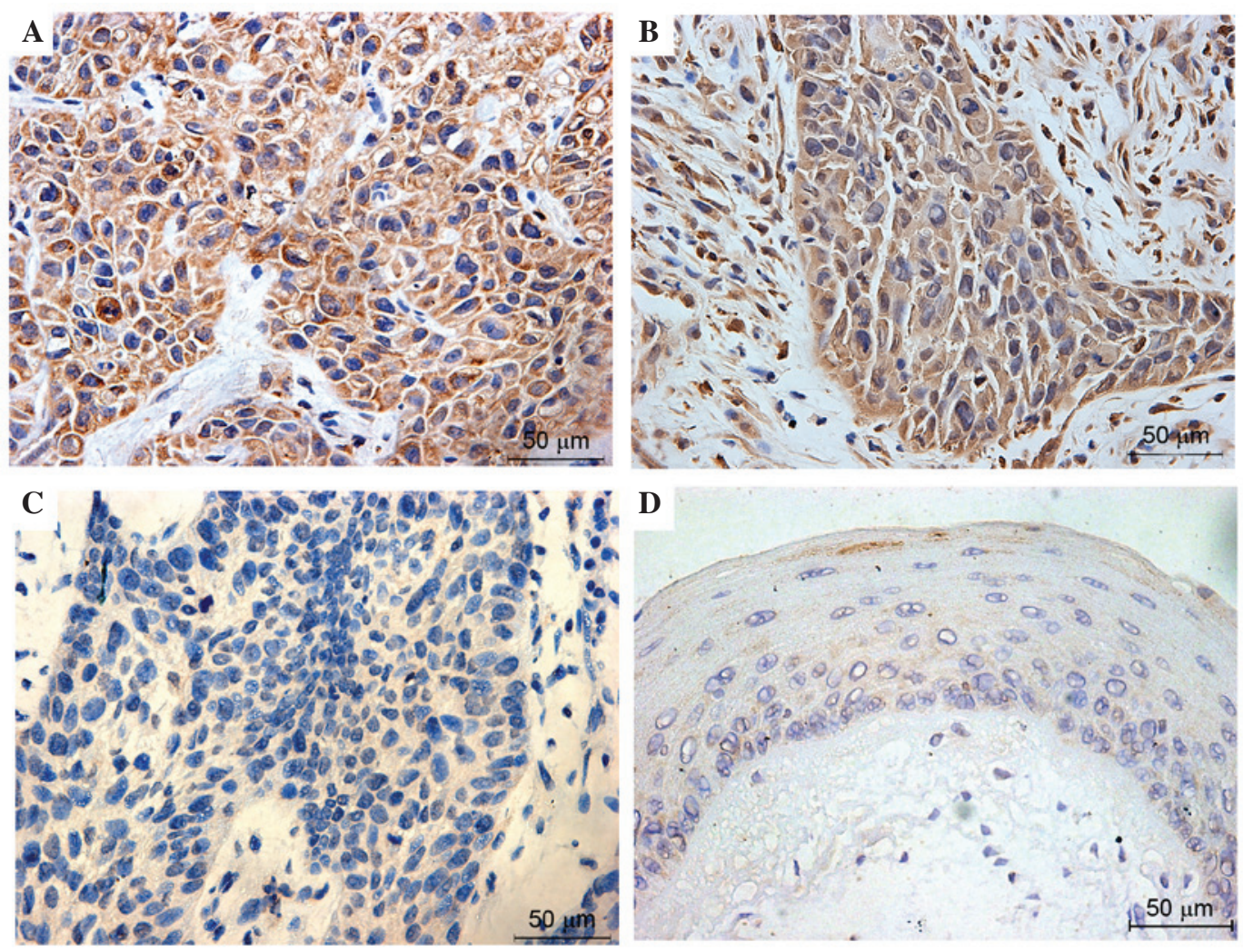

Figure 4. Representative immunohistochemical staining of HO-1 in laryngeal squamous cell carcinoma and adjacent normal mucosa. The top two images exhibit (A) strong and (B) positive expression of HO-1, which was primarily observed in the cancer cell cytoplasm. The bottom two pictures exhibit negative HO-1 expression in (C) laryngeal cancer and (D) pericarcinomatous tissues. Normal mucosa produced almost no positive HO-1 expression. Positive cytoplasmic HO-1 was defined as a score $\geq 0.5$ (magnification $x 40$ ). HO-1, heme oxygenase-1.

KEAP1, NQO-1 and HO-1 were predominantly observed in the cytosol of tumor cells, and these proteins were also highly expressed in majority of laryngeal cancer tissues. Overall, the expression levels of NRF2, KEAP1, NQO-1 and HO-1 were very low or negative in the most normal tissues. Figs. 1-4 present the representative expression of NRF2, KEAP1, NQO-1 and HO-1 in laryngeal cancer and pericarcinomatous normal tissues.

Semiquantitative scoring analyses of immunohistochemical staining (Fig. 5A) demonstrated that the majority of normal larynx mucosa exhibited negative or weak expression of NRF2, KEAP1, NQO-1 and HO-1. By contrast, the expression of these proteins was markedly enhanced to varying degrees in advanced laryngeal cancer tissues. Positive staining of nuclear NRF2 (immunoscore $\geq 0.1$ ) was observed in 26 cases of laryngeal cancer (79\%). Of 33 patient tumors, 17 tumors (52\%) exhibited moderate-strong nuclear accumulation $(\geq 2.0)$. However, only 4 adjacent normal specimens (12\%) exhibited weak NRF2 staining. There was a significant difference in the expression of NRF2 protein between the tumor and normal tissues $(\mathrm{P}<0.01)$. KEAP1-positive immunostaining (immunoscore $\geq 0.5$ ) was observed in 23 cancer samples (70\%), of which 14 cases $(42 \%)$ exhibited moderate-strong KEAP1 expression $(\geq 2.0)$. The positive rate was significantly higher in cancerous tissues compared with normal tissues $(5 / 33,15 \%)$ $(\mathrm{P}=<0.01)$. The expression patterns of NQO-1 and HO-1 in all of the laryngeal cancer and normal specimens were similar. Of the cancerous tissues, 25 (76\%) and 27 (82\%) samples exhibited
NQO-1- and HO-1-positive staining. This was a significant increase compared with the neighboring non-tumor specimens $(\mathrm{P}<0.01)$.

Immunoblot analyses were also performed to compare the expression of NRF2, KEAP1, NQO-1 and HO-1 in laryngeal cancer and the paired normal tissues (Fig. 5B). Western blot analyses demonstrated that the protein expression levels of NRF2, KEAP1, NQO-1 and HO-1 were markedly increased in advanced cancerous tissues compared to with the adjacent normal tissues.

The Pearson correlation coefficient was chosen to represent associations between the expression of nuclear NRF2 with the expression of regulatory protein KEAP1 and the phase II detoxifying enzymes, NQO-1 and HO-1, to determine the biological effect of KEAP1/NRF2 system in patients with advanced laryngeal cancer. High KEAP1, NQO-1 and HO-1 expression in laryngeal cancer tissues were all statistically correlated with nuclear NRF2 $(\mathrm{P}=0.0033, \mathrm{r}=0.4966$; $\mathrm{P}=0.0021, \mathrm{r}=0.5163$; and $\mathrm{P}=0.0361, \mathrm{r}=0.3662$; respectively; Fig. 6A and B). However, no significant correlation between the expression of KEAP1 and NQO-1, and HO-1 (Fig. 6C) was identified in advanced laryngeal carcinoma. No significant correlation was identified between NQO-1 and HO-1 expression levels ( $\mathrm{P}=0.165 ; \mathrm{r}=0.24750$; Fig. $6 \mathrm{D})$.

Additionally, the association between the expression of KEAP1/NRF2 and clinicopathological features in advanced laryngeal cancer tissues. It was revealed that the staining of NRF2, KEAP1, NQO-1 and HO-1 was independent of age, 


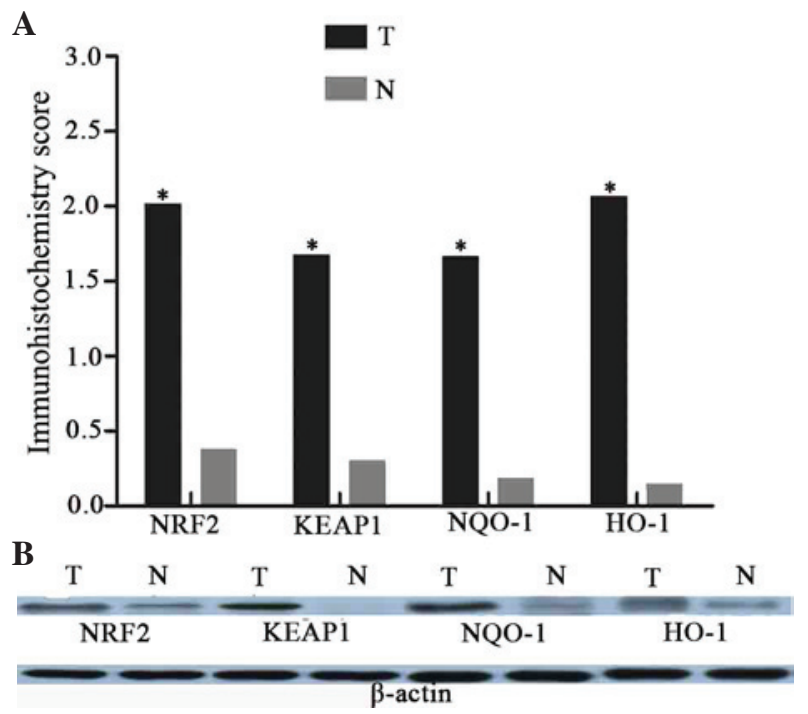

Figure 5. The expression of NRF2, KEAP1, NQO-1 and HO-1 proteins in laryngeal cancer and adjacent normal tissues. (A) Mean immunostaining scores of KEAP1, NRF2, NQO-1 and HO-1. There are significant differences between laryngeal cancer and adjacent normal tissues on these four proteins expression ( $\mathrm{P}<0.01$, T vs. N). (B) Immunoblot detection of KEAP1, NRF2, NQO-1 and HO-1 demonstrates that the protein levels are observably increased in tumor samples, compared with the normal tissues. T, laryngeal cancer; N, normal tissues; NRF2, nuclear factor (erythroid-derived 2)-like 2; KEAP1, kelch-like ECH-associated protein-1; NQO-1, quinone oxidoreductase-1; HO-1, heme oxygenase-1.

lymph node status, tumor stage (clinical stage III and IV) and the tumor size (Fig. 7).

\section{Discussion}

The pathogenesis and etiology of laryngeal carcinoma is not clear. However, numerous studies demonstrated that oxidative stress directly or indirectly causes DNA damage, which is a predisposing factor to malignant lesions $(14,15)$. The body has established appropriate self-defense mechanisms to cope with these harmful stimuli, including the KEAP1-NRF2-AREs pathway (16). KEAP1 was discovered in 1999 (17), and the KEAP1 system is constantly researched, particularly NRF2. However, specific research on the association between laryngeal cancer and this pathway is limited, despite certain notable recent advances (18). Little is understood about oxidative stress and the activation of carcinogenesis pathways in laryngeal cancer. The clinical value of the NRF2, KEAP1, NQO-1 and HO-1 in advanced laryngeal cancer was investigated in the current study.

NRF2 is a critical gene that with a core role in KEAP1/NRF2 system, and NRF2 expression is associated with poor prognosis in patients with cancer. NRF2 protects normal cells, however it also promotes the survival of cancer cells (19). As a result, NRF2 has been well studied. The present study evaluated the expression levels and correlation of KEAP1, NRF2, NQO-1 and HO-1, and their associations with clinicopathological features in advanced laryngeal cancer tissue. The results of the current study demonstrated that KEAP1, NRF2, NQO-1 and HO-1 were significantly increased in advanced laryngeal carcinoma samples compared with the normal tissues. Notably, in tumor samples NRF2 was primarily localized in nuclei, and KEAP1, NQO-1 and HO-1 were principally expressed in the cytoplasm. These results were consistent with previous research. Stacy et al (20) reported that $91.5 \%$ of head and neck tumor tissues (43/47) exhibited a statistically significant increase in NRF2 expression compared with normal mucosa. The positive rate of nuclear NRF2 was higher in the previous study than in the present study, however the previous study included other cancer tissues in addition to larynx, and no separate analysis for NRF2 expression in laryngeal carcinoma was performed. Stacy et al (20) also detected a high expression of KEAP1 in the cytoplasm of tumor cells. An analysis of the association between KEAP1 and NRF2 revealed that KEAP1 was not negatively associated with nuclear NRF2 (20). Another study reported the significant association of KEAP1 expression with nuclear NRF2 in oral squamous cell carcinoma (21). This observation was verified in head and neck cancers and non-small cell lung carcinomas (NSCLC), in which nuclear NRF2 positivity was as high as $61.8 \%$ and its expression was statistically associated with higher KEAP1 expression (22). The frequency of positive nuclear NRF2 was significantly higher in pulmonary squamous cell carcinomas than adenocarcinomas, however, KEAP1 exhibited the opposite expression pattern. However, the present study only analyzed laryngeal squamous cell carcinoma because adenocarcinoma of the larynx presents at an extremely low incidence in the clinic. Nuclear NRF2 overexpression is also observed in serous cystadenocarcinomas, with a positive rate of $71.1 \%$ (27/38) (23), which is the same level as the results of the current study in laryngeal carcinoma. The positive expression of nuclear NRF2 was $>30 \%$ in one report of pancreatic adenocarcinoma, however, cytoplasmic NRF2 expression accounted for $86 \%$, which is higher that the results of the present study (24). The disparity with the results of the present study may be due to the different tumor tissues used.

Various recent studies suggest that KEAP1 mutations lead to constitutive activation and the nuclear accumulation of NRF2, which enhances the expression of antioxidative and detoxifying enzymes $(25,26)$. Certain data demonstrated that KEAP1 mutations occur widely in solid cancers, irrespective of histological type (27). Unfortunately, a sequence analysis of KEAP1 was not conducted in the current study. However, we speculate that this mechanism may be pervasive in laryngeal cancer, and it may also partially explain the positive, rather than negative, KEAP1 correlation with nuclear NRF2 in laryngeal carcinoma tissues. Genetic mutations may give rise to KEAP1 dysfunction, which leads to the nuclear accumulation of NRF2 and prompts the expression of downstream proteins. Although the mechanism of NRF2 nuclear transfer in laryngeal cancer tissues is not known, the current study demonstrated that high levels of nuclear NRF2 protein were significantly associated with KEAP1, NQO-1 and $\mathrm{HO}-1$ overexpression $(\mathrm{P}=0.0033, \mathrm{r}=0.4966 ; \mathrm{P}=0.0021$, $\mathrm{r}=0.5163$ and $\mathrm{P}=0.0361, \mathrm{r}=0.3662$; respectively) and the expression of detoxification and antioxidant enzymes NQO-1 and HO-1 was markedly elevated in cancer tissue compared with normal mucosa, which is consistent with previous reports in gallbladder cancer and NSCLC (28). These results revealed that NRF2, KEAP1, NQO-1 and HO-1 may be part of a signaling pathway and have important biological effects on the occurrence and development of laryngeal cancer. 

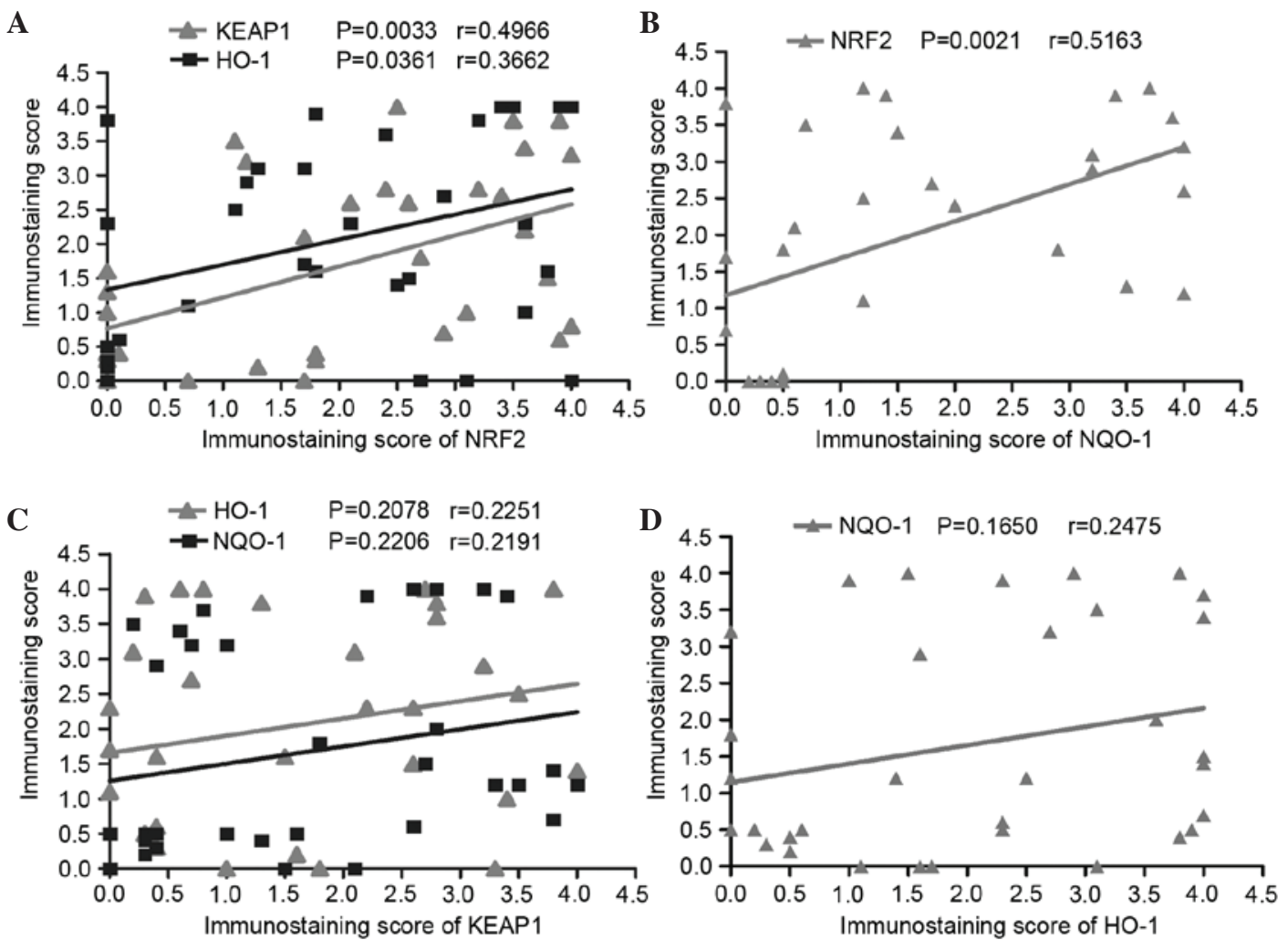

Figure 6. Correlation of KEAP1 and NRF2 with NQO-1 and HO-1 in laryngeal cancer tissues using the Pearson correlation coefficient test. The expression of NRF2 is closely correlated with (A) KEAP1 $(\mathrm{P}=0.0033, \mathrm{r}=0.4966)$ and HO-1 $(\mathrm{P}=0.0361, \mathrm{r}=0.3662)$ and $(\mathrm{B}) \mathrm{NQO}-1(\mathrm{P}=0.0021, \mathrm{r}=0.5163)$. The expression of KEAP1 has no significant correlation with $(\mathrm{C}) \mathrm{NQO}-1(\mathrm{P}=0.2206)$ and HO-1 $(\mathrm{P}=0.2078)$ and $(\mathrm{D})$ there is no significant relationship between the expression of HO-1 and NQO-1 in advanced laryngeal cancer tissues ( $\mathrm{P}=0.1650)$. KEAP1, kelch-like ECH-associated protein-1; HO-1, heme oxygenase-1; NRF2, nuclear factor (erythroid-derived 2)-like 2; NQO-1, quinone oxidoreductase-1.

A

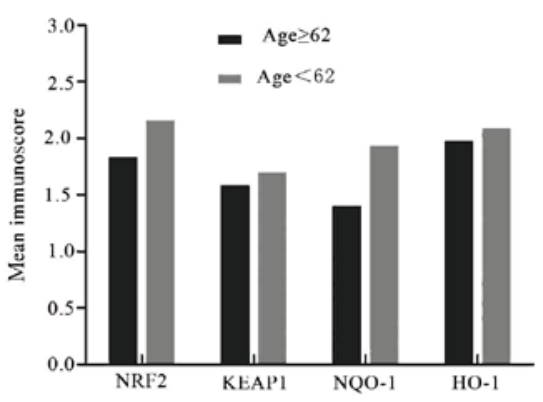

C

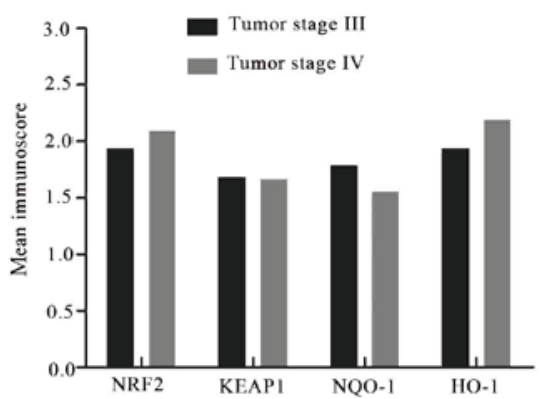

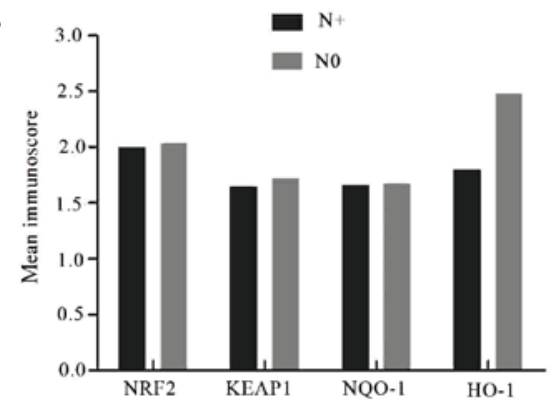

D

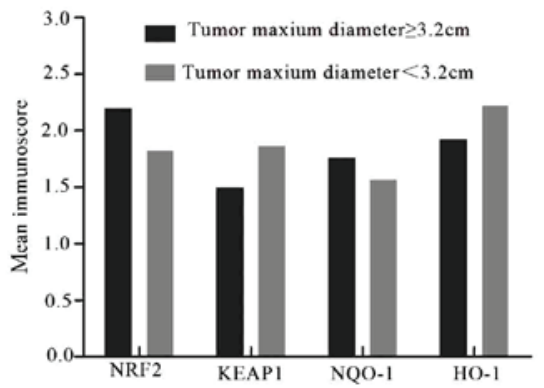

Figure 7. Association between the expression NRF2, KEAP1, NQO-1 and HO-1 and clinicopathological features in advanced laryngeal cancer tissues. (A) The expression levels of NRF2, KEAP1, NQO-1 and HO-1 in the patients with age $<62$ years old are not significantly higher than the patients with age $\geq 62$ ( $\mathrm{P}=0.157,134,574$ and 0.200 , respectively). (B) The expression levels of these proteins are not obviously associated with lymph node metastasis ( $\mathrm{P}=0.867$, $0.926,0.957$ and 0.266 respectively). (C) There are no significant differences in the expression levels of NRF2, KEAP1, NQO-1 and HO-1 between the patients with diagnosed tumor stage III and IV ( $\mathrm{P}=0.761,0.972,0.667$ and 0.621 respectively). (D) Tumor size has no significant impact on the expression levels of NRF2, KEAP1, NQO-1 and HO-1 ( $\mathrm{P}=0.531,0.809,0.315$ and 0.828 respectively). Data are expressed as the mean values of patient characteristics. $\mathrm{N}+$, lymph node metastasis; N0, no lymph node metastasis; NRF2, nuclear factor (erythroid-derived 2)-like 2; KEAP1, kelch-like ECH-associated protein-1; NQO-1, quinone oxidoreductase-1; HO-1, heme oxygenase-1. 
However, due to the relatively small sample size in the current study, it is necessary to enlarge the sample numbers and conduct further research. Western blotting analyses also confirmed that NRF2, KEAP1, NQO-1 and HO-1 were markedly elevated in laryngeal cancer tissue compared with adjacent normal tissue, which exhibited low expression. Unfortunately, could not be obtained for western blotting analyses of nuclear NRF2 expression. However, total NRF2 protein in laryngeal cancer tissues was markedly higher than in normal tissues.

Reports regarding the association between the expression of KEAP1/NRF2 system and clinicopathological features are not consistent. A previous study reported that $\mathrm{Nrf} 2$ and HO-1 expression was associated with tumor stage and metastasis, and not associated with age in gallbladder cancer (28), whereas in NSCLC no significant correlation is observed. Liao et al (23) demonstrated that NRF2 expression is unrelated to age and lymph node metastasis in ovarian epithelial carcinoma. Furthermore, it was reported that KEAP1 and NRF2 were not significantly correlated with tumor stage and lymph node status in oral squamous cell carcinoma, which supports the results of the current study $(21-23,28)$. The results of the present indicate that the expression of NRF2, KEAP1, NQO-1 and HO-1 is independent of age, tumor stage and lymph node status. Additionally, the association between their expression and tumor size was analyzed and revealed to not be significant. It is suggested that their increased expression was common in advanced laryngeal cancer and further investigations are necessary to confirm this conclusion.

This report is the first to specifically analyze the expression levels and correlation of NRF2, KEAP1, NQO-1 and HO-1, and their association with clinicopathological features in advanced laryngeal cancer. The present study confirmed that the expression of NRF2, KEAP1, NQO-1 and HO-1 are increased significantly in advanced laryngeal squamous cell carcinoma, compared with the adjacent normal mucosa. Remarkable relevance exists between high expression of KEAP1, NQO-1, HO-1 and nuclear NRF2. Additionally, their expression levels were independent of age, tumor stage (clinical stage III and IV), tumor size and lymph node metastasis. These results suggest that increased expression of NRF2, KEAP1, NQO-1 and HO-1 were common and may possess important clinical value to diagnose and treat advanced laryngeal cancer.

\section{Acknowledgements}

The project was supported by the Science and Technology Commission of Shanghai Municipality (grant no. KW12010), Health Bureau of New Pudong District (grant no. PW2012D-4) and Shanghai Municipal Education Commission (grant no. 13ZZ008).

\section{References}

1. Harrington KJ, Nutting CM and Pandha HS: Gene therapy for head and neck cancer. Cancer Metastasis Rev 24: 147-164, 2005.

2. Siegel RL, Miller KD and Jemal A: Cancer statistics, 2015. CA Cancer J Clin 65: 5-29, 2015.

3. Ferlay J, Shin HR, Bray F, Forman D, Mathers C and Parkin DM: Estimates of worldwide burden of cancer in 2008: GLOBOCAN 2008. Int J Cancer 127: 2893-2917, 2010.
4. American Society of Clinical Oncology, Pfister DG, Laurie SA, Weinstein GS, Mendenhall WM, Adelstein DJ, Ang KK, Clayman GL, Fisher SG, Forastiere AA, et al: American Society of Clinical Oncology clinical practice guideline for the use of larynx-preservation strategies in the treatment of laryngeal cancer. J Clin Oncol 24: 3693-3704, 2006.

5. Cho HY, Reddy SP and Kleeberger SR: Nrf2 defends the lung from oxidative stress. Antioxid Redox Signal 8: 76-87, 2006.

6. Inci E, Civelek S, Seven A, Inci F, Korkut N and Burçax G: Laryngeal cancer: In relation to oxidative stress. Tohoku J Exp Med 200: 17-23, 2003

7. Jaramillo MC and Zhang DD: The emerging role of the Nrf2-Keap1 signaling pathway in cancer. Genes Dev 27: 2179-2191, 2013.

8. Singh A, Boldin-Adamsky S, Thimmulappa RK, Rath SK, Ashush H, Coulter J, Blackford A, Goodman SN, Bunz F, Watson WH, et al: RNAi-mediated silencing of nuclear factor erythroid-2-related factor 2 gene expression in non-small cell lung cancer inhibits tumor growth and increases efficacy of chemotherapy. Cancer Res 68: 7975-7984, 2008.

9. Khor TO, Huang MT, Prawan A, Liu Y, Hao X, Yu S, Cheung WK, Chan JY, Reddy BS, Yang CS and Kong AN: Increased susceptibility of NRF2 knockout mice to colitis-associated colorectal cancer. Cancer Prev Res (Phila) 1: 187-191, 2008.

10. Edge SB, Compton CC and The American Joint Committee on Cancer: The 7th edition of the AJCC cancer staging manual and the future of TNM. Ann Surg Oncol 17: 1471-1474, 2010.

11. Li CJ, Wang SZ, Wang SY and Zhang YP: Assessment of the effect of local application of amifostine on acute radiation-induced oral mucositis in guinea pigs. J Radiat Res 55: 847-854, 2014.

12. Manne U, Myers RB, Moron C, Poczatek RB, Dillard S, Weiss H, Brown D, Srivastava S and Grizzle WE: Prognostic significance of Bcl-2 expression and p53 nuclear accumulation in colorectal adenocarcinoma. Int J Cancer 74: 346-358, 1997.

13. Piyathilake CJ, Bell WC, Oelschlager DK, Heimburger DC and Grizzle WE: The pattern of expression of $\mathrm{Mn}$ and $\mathrm{Cu}-\mathrm{Zn}$ superoxide dismutase varies among squamous cell cancers of the lung, larynx, and oral cavity. Head Neck 24: 859-867, 2002.

14. Han Y and Chen JZ: Oxidative stress induces mitochondrial DNA damage and cytotoxicity through independent mechanisms in human cancer cells. Biomed Res Int 825065, 2013.

15. McLean LS, Watkins CN, Campbell P, Zylstra D, Rowland L, Amis LH, Scott L, Babb CE, Livingston WJ, Darwanto A, et al: Aryl hydrocarbon receptor ligand 5F 203 induces oxidative stress that triggers DNA damage in human breast cancer cells. Chem Res Toxicol 28: 855-871, 2015.

16. Dwivedi R, Raturi D, Kandpal N, Dwivedi R, Singh R and Puri V: Oxidative stress in patients with laryngeal carcinoma. Indian J Cancer 45: 97-99, 2008.

17. Dinkova-Kostova AT, Holtzclaw WD and Kensler TW: The role of Keap1 in cellular protective responses. Chem Res Toxicol 18: 1779-1791, 2005.

18. Kensler TW and Wakabayashi N: Nrf2: Friend or foe for chemoprevention? Carcinogenesis 31: 90-99, 2010.

19. Lee JM and Johnson JA: An important role of NRF2-ARE pathway in the cellular defense mechanism. J Biochem Mol Biol 37: 139-143, 2004.

20. Stacy DR, Ely K, Massion PP, Yarbrough WG, Hallahan DE, Sekhar KR and Freeman ML: Increased expression of nuclear factor E2 p45-related factor 2 (NRF2) in head and neck squamous cell carcinomas. Head Neck 28: 813-818, 2006.

21. Huang CF, Zhang L, Ma SR, Zhao ZL, Wang WM, He KF, Zhao YF, Zhang WF, Liu B and Sun ZJ: Clinical significance of KEAP1 and NRF2 in oral squamous cell carcinoma. PLoS One 8: e83479, 2013.

22. Solis LM, Behrens C, Dong W, Suraokar M, Ozburn NC, Moran CA, Corvalan AH, Biswal S, Swisher SG, Bekele BN, et al: Nrf2 and Keap1 abnormalities in non-small cell lung carcinoma and association with clinicopathologic features. Clin Cancer Res 16: 3743-3753, 2010.

23. Liao H, Zhou Q, Zhang Z, Wang Q, Sun Y, Yi X and Feng Y: NRF2 is overexpressed in ovarian epithelial carcinoma and is regulated by gonadotrophin and sex-steroid hormones. Oncol Rep 27: 1918-1924, 2012.

24. Soini Y, Eskelinen M, Juvonen P, Kärjä V, Haapasaari KM, Saarela A and Karihtala P: Nuclear Nrf2 expression is related to a poor survival in pancreatic adenocarcinoma. Pathol Res Pract 210: 35-39, 2014. 
25. Ohta T, Iijima K, Miyamoto M, Nakahara I, Tanaka H, Ohtsuji M, Suzuki T, Kobayashi A, Yokota J, Sakiyama T, et al: Loss of Keap1 function activates Nrf2 and provides advantages for lung cancer cell growth. Cancer Res 68: 1303-1309, 2008.

26. Konstantinopoulos PA, Spentzos D, Fountzilas E, Francoeur N, Sanisetty S, Grammatikos AP, Hecht JL and Cannistra SA: Keap1 mutations and Nrf2 pathway activation in epithelial ovarian cancer. Cancer Res 71: 5081-5089, 2011.
27. Yoo NJ, Kim HR, Kim YR, An CH and Lee SH: Somatic mutations of the KEAP1 gene in common solid cancers. Histopathology 60: 943-952, 2012

28. Wang J, Zhang M, Zhang L, Cai H, Zhou S, Zhang J and Wang Y: Correlation of Nrf2, HO-1, and MRP3 in gallbladder cancer and their relationships to clinicopathologic features and survival. J Surg Res 164: e99-e105, 2010. 